\title{
Del determinismo de la duración a la apertura del instante: propuestas ante el pensamiento ambiental evolucionista
}

\author{
From determining the moment duration to instant starting \\ point: propositions for evolutionary environmentalist \\ viewpoints
}

Antonio de LISIO*

\section{RESUMEN}

En este trabajo se intenta plantear alternativas ante la lógica de la duración evolutiva como marco temporal predominante en la convencionalidad ambientalista. Frente a la racionalidad evolutiva que obliga al determinismo de la causa original bien cósmica o bien planetaria, se plantea axiomáticamente el alcance de una lógica alternativa de los "instantes" epistemológicos, propiciada por la interpretación policéntrica del ambiente. En función del planteamiento de esta apertura de base frente al determinismo de lo primigenio, se derivan otras dos propuestas más operativas vinculadas al abordaje autoreferencial del espacio-tiempo de las organizaciones ambientales en el marco de la termodinámica no lineal o de los sistemas abiertos alejados del equilibrio. Finalmente se plantean algunas repercusiones epistemológicas de las propuestas desarrolladas, tratando especialmente de superar algunas visiones simplificadoras del discurso retórico ambientalista.

Palabras-clave: duración, instante, policentrismo, entropía, neguentropía

\begin{abstract}
In this paper, some alternatives are suggested while dealing with the logic of an evolutionary duration as a predominant timing in the environmentalist convention. Due to the fact that people are facing an evolutionary rationality which demands determinism to the cosmical original or planetary cause ,all are expected to deal with an alternative logic to "epistomological moments" It is propelled by a policentric interpretation of the moment. From this approach concerning the basic opening while facing the originary determininsm, two proposals are offered and related to self-reference of environmentalist organization's timing and space which refer to non-linear or thermodynamic or to open systems which are not balanced. Finally, some epístemological repercussions of the developed proposals are approached in order to find a way to overcome some simplistic views of the environmentalist rethorical speech.

Key-words: duration, instant, policentrism, entropy, non-entropy
\end{abstract}

* Cenamb - Universidad Central de Venezuela. 
LISIO, A. de. Del determinismo de la duración a la apertura del instante...

\section{Introducción}

El tiempo se ha convertido en la dimensión que mejor permite entender las insuficiencia del método de la ciencia convencional: muy capaz en la demostración pero muy limitada en el descubrimiento. A pesar de que lo ambiental ha tratado de convertirse en un planteamiento alternativo, en la práctica el ambientalismo se ha subsumido en la convencionalidad científica cartesiana. Más allá de los actos de fe de una cierta retórica del discurso holístico maximalista, la práctica ambientalista se ha visto predominantemente orientada por los postulados básicos del método cartesiano. De manera particular debe destacarse como ejemplo de esta contradicción entre el discurso y la práctica el caso de las posturas evolutivas que primordialmente se defienden, en el tipo de ambientalismo, que al largo de este trabajo calificaremos de convencional. Los ambientalistas evolutivos, defienden la necesidad operativa de remontar a una causa original primigenia, para derivar posteriormente a un conjunto de consecuencias.

El método cartesiano fuerza a remontar de causa en causa hacia una causalidad inicial, situada evidentemente en el pasado, para de esta manera aislar parcelas causa/ efecto, tratando posteriormente de generalizar resultados. Es así como se llega a otro fundamento de la ciencia convencional: la universalidad del conocimiento. La generalización siempre es posible cuando se actúa sobre una totalidad seccionada que reduce la complejidad de los procesos.

\section{FIGURA 1 - EL IDEOGRAMA AMBIENTAL}

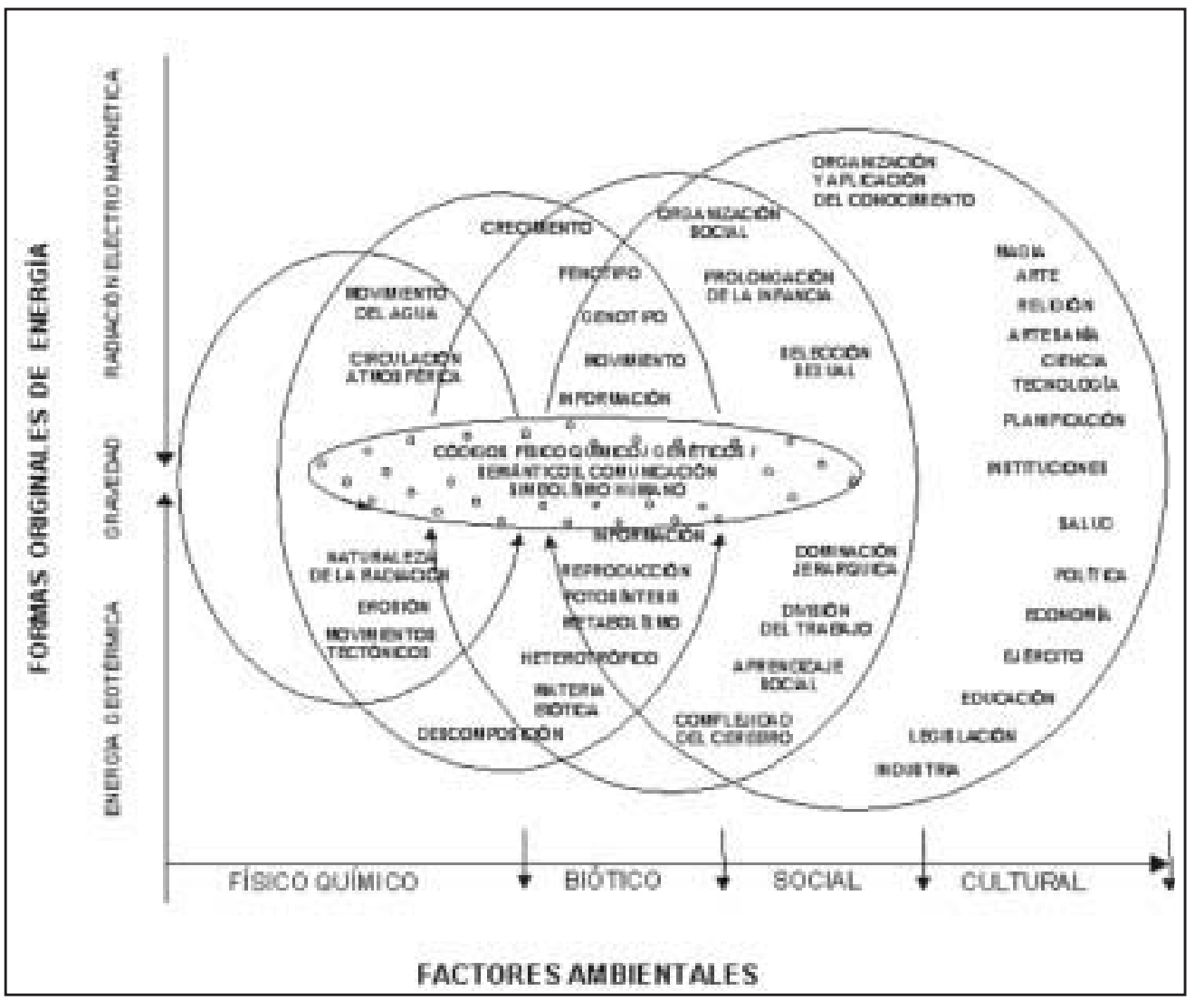

FUENTE: Gonzáles, 1984. Elaboración y reformulación propia. 
De acuerdo a De Rosnay,

...para el descubrimiento clásico la acción libre aparece como imposible en el plano científico (teoría de la conciencia epifenoménica), mientras que la observación es obvia. Es que existen dos modos fundamentales de actividad de la conciencia, uno corresponde a la transformación de la neguentropia en información. Es el proceso de la observación-información científicaadquisición de conocimiento. El otro corresponde a la transformación inversa de información en neguentropia. El primer proceso es el de la actualización de lo adquirido. De la difusión de la reproducción y la duplicación. Por el contrario el proceso inverso, el de la acción creadora, cuesta muy caro en informaciones. Por ello la creación de un original (a diferencia de la duplicación) parece tan difícil. (DE ROSNAY, 1976, p. 89)

\section{La duración evolutiva: el determinismo del continuidad naturaleza-cultura}

La visión evolutiva en el ambiente descansa en el fundamento de la necesidad de comprender al hombre y su cultura como una derivación de la naturaleza. Este principio incuestionable a nivel ontológico, desde el punto de vista epistemológico y metodológico, introduce serias dificultades para comprender al ambiente como conjunto de factores diversos, que son producto de las emergencias, que se han ido superponiendo a la primigenia realidad físico-química. Para el análisis de esta visión, se ha considerado conveniente centrarse en la evaluación del ideograma ambiental (figura 1), uno de los intentos mejor logrados como expresión de esta orientación.

La lectura convencional que hasta ahora se ha hecho en el ideograma puede ser definida como "evolutiva planetaria”, es decir partiendo de los factores físicos se determinan tanto las interrelaciones intranivélicas (a nivel de lo físico-químico o protobiótico) como internivélicos (interrelaciones con los niveles restantes bióticos, social y cultural (figura 2). De tal manera que los procesos y transformaciones que se estudian en el marco de esta interpretación tienden a favorecer la dimensión evolutiva, - por lo general expresada en términos de antes y después en la consideración de los diferentes componentes que estructuran el ambiente. Esta es una visión que anclada en la ecología evolutiva, exige partir de los determinantes que han regulado la propagación de la energía solar en el planeta. Para ello resulta fundamental la comprensión de la manera como los tres tipos de rayos solares (actínicos, ultravioletas e infrarrojos), se transmiten en los distintos medios y substratos, es decir, lo que rodea al organismo de manera inmediata y las superficies y materiales sólidos sobre o dentro de los cuales viven los organismos que expresan la naturaleza de lo protobiótico: agua, aire, tierra. De allí que, como procesos clave de entrada para el ideograma, aparezcan aquellos que dan cuenta de las interrelaciones de la energía electromagnética sobre la materia protobiótica. En especial se deben recalcar los códigos físico-químicos, los cuales, en esta interpretación evolutiva-planetaria del ambiente, se utilizan para iniciar un encadenamiento energético que posteriormente conduce consecutivamente a la vida, la sociedad y la cultura. Estos códigos son la llave de entrada al conjunto de los factores físico-químicos que actúan como:

- generadores, transmisores y reproductores de las diferentes formas de fuerzas existentes en el ambiente;

- sustrato químico para el desarrollo de los procesos vitales fuentes y medios para la propagación de estímulos sensoriales (GONZÁLEZ et al., 1984, p. 16).

De esta forma los componentes físico-químicos se convertirían en el primer eslabón de un continuum que conducen del sol y el sistema planetario a la cultura humana; de las reacciones del átomo de helio a la poesía, en una lectura que obligatoriamente se realiza en la flecha del devenir de la energía solar en el planeta, es decir un recorrido que abarca más de cuatro mil quinientos millones de años. En este sentido esta interpretación del ambiente sigue la tendencia de otros intentos como "el 'Ambiente-Mundo' de D. Vidart (1986) y la Boule de fleches de Danserau (1976), que se sustentan en la lógica de la duración, de la permanencia, mediante la cual pareciera que lo inmanentemente ambiental está supeditado a una causa primera físico-química, bien de tipo general cosmológico o bien particular del planeta Tierra". En estes tipos de interpretaciones basadas en la noción de duración como extensión en el tiempo, el acento se pone en los aspectos que muestran el recorrido completo de la evolución de la materia en el planeta. Sin embargo, no siempre para explicar los hechos se debe remontar al origen del proceso, por cuanto se estaría propiciando una visión determinista desde el origen, en la que las situaciones una vez iniciadas 
LISIO, A. de. Del determinismo de la duración a la apertura del instante...

FIGURA 2 - EL POLICENTRISMO AMBIENTAL. LECTURA DE SDE LO FÍSICO QUÍMICO

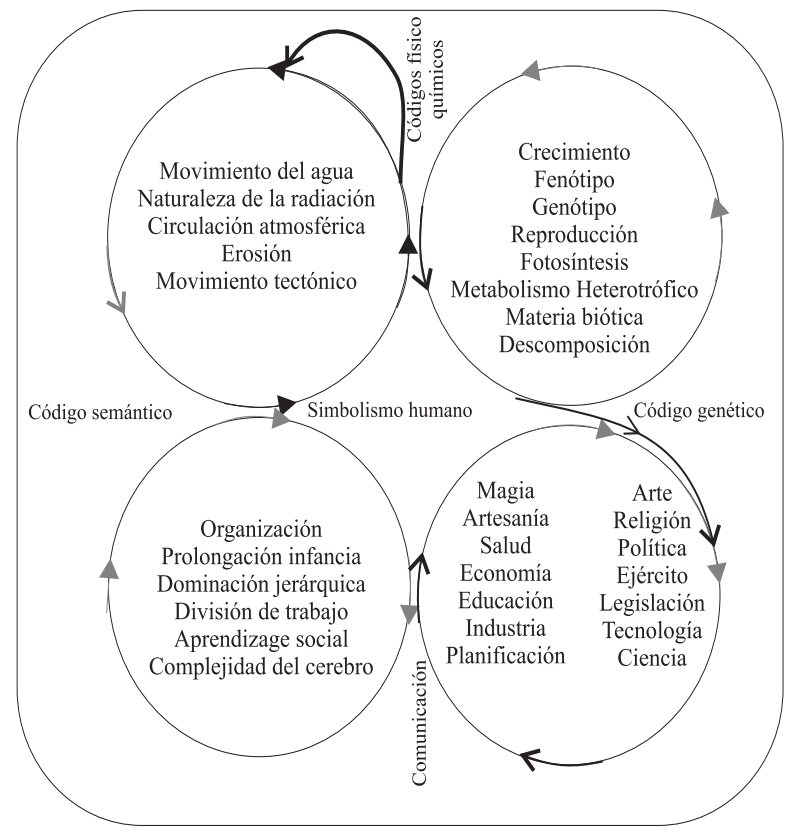

FIGURA 3 - EL POLICENTRISMO AMBIENTAL. LECTURA DE SDE LO BIÓTICO

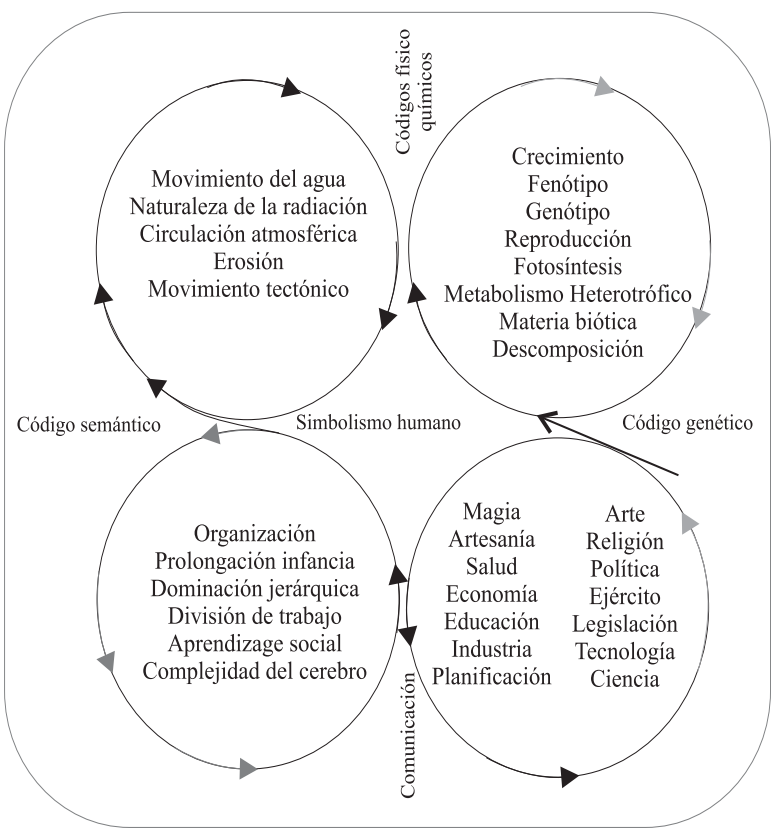

FIGURA 4 - EL POLICENTRISMO AMBIENTAL. LECTURA DE SDE LO SOCIAL

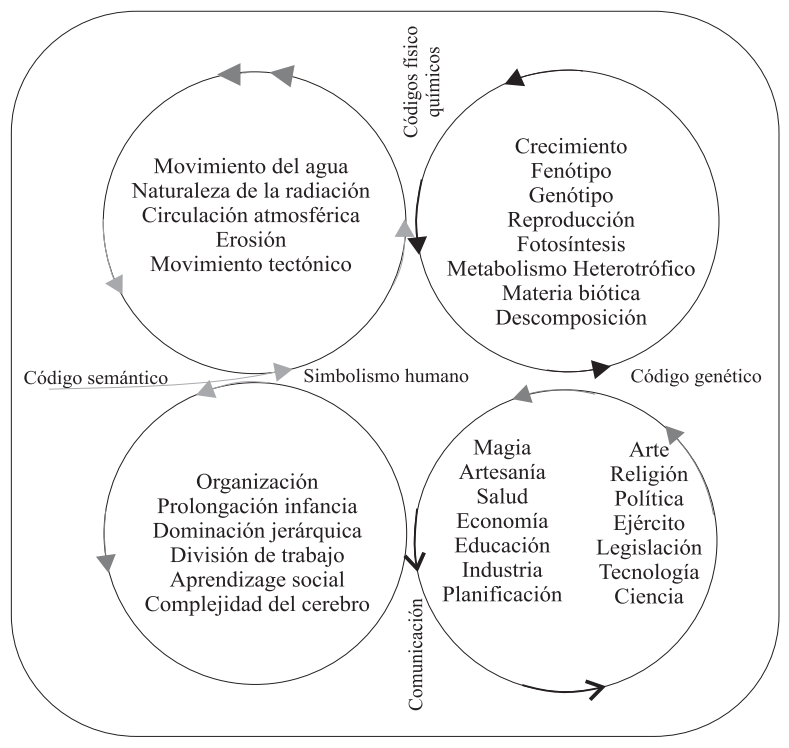

FIGURA 5 - EL POLICENTRISMO AMBIENTAL. LECTURA DE SDE LO CULTURAL

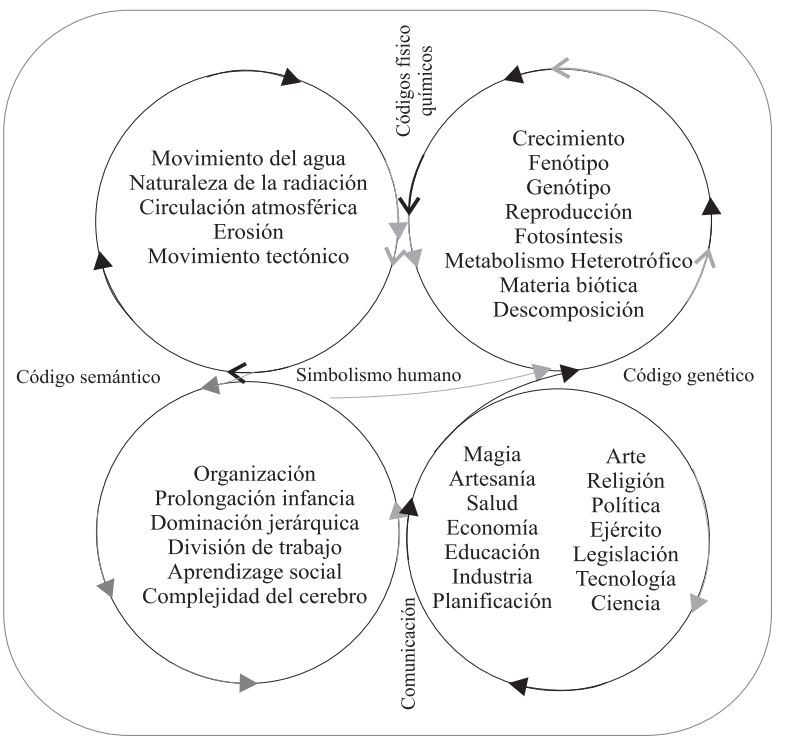


parecieran no estar sujetas a eventuales modificaciones y cambios.

Contrariamente a la luz de la termodinámica no lineal - la que se aplica para el estudio de los sistemas en la realidad y no en las condiciones experimentales controladas en laboratorios (PRIGOGINE; STENGER, 1977; 1988) - se considera que cualquier unidad ambiental al estar abierta a los intercambios energéticos con sus contextos de referencia, se caracteriza por procesos no siempre lineales. De aceptarse este planteamiento, la linealidad evolutiva se convertiría solo en una posibilidad, más no en una constante. En las interpretaciones evolutivas, se ha pretendido convertir al ambiente en una suma de encadenamientos lineales, reduciendo la capacidad de interpretación de las emergencias y bifurcaciones que han permitido la emergencia de la vida de la physis del planeta, de la sociedad de la biología y de la cultura de la sociedad. Si se acepta a los niveles del ambiente físico-químico, biológico, social y cultural como una sucesión de emergencias, donde cada uno así como expresa características propias del nivel anterior también manifiesta las propias que permiten su identificación como una diversidad en la unidad ambiental, la lectura centrada en lo físico-químico no puede considerarse como única.

Circunscribiéndonos al caso del ideograma más que una lectura lineal se debería tratar de propiciar su interpretación como holografía, es decir, una visión en la que se pueda captar desde cualquier nivel la diversidad y complejidad del conjunto de factores ambientales. Más que holismo maximalista - es decir, sin valorar el carácter de las partes - se requiere de capacidad holográfica de interpretación holística minimalista - es decir reconociendo la importancia de las partes en la constitución del todo ambiental.

A la lectura convencional evolutiva y lineal del ideograma, en el marco de una visión holográfica- holísticaminimalista, se le pueden anteponer otras que expresen recorridos que deben ser iniciados desde los otros "instantes" identificados en la estructura tetracéntrica de los cuatros niveles considerados.

Es así como centrados en el nivel de lo biótico, y atendiendo a la naturaleza energética del ideograma, se puede utilizar el código genético para lograr una interpretación "evolutiva viviente" del esquema (figura 3). De manera similar se pueden realizar lecturas iniciadas desde lo social partiendo del código semántico y desde lo cultural iniciando el simbolismo humano (figura 4 y 5, respectivamente).

En estas lecturas alternativas del ideograma, se pasa del peso de la fuerza y la materia al de la información (figura 6), reconociéndose fundamentalmente el papel de los códigos de información genéticos y semánticos como factores clave de la dinámica ambiental.

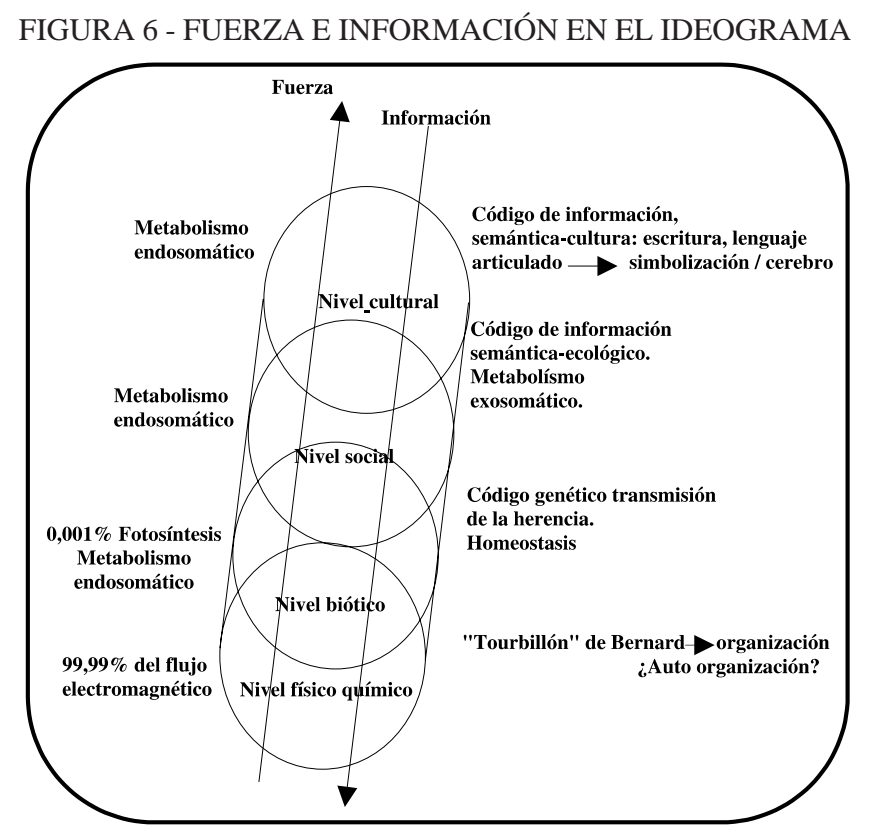


LISIO, A. de. Del determinismo de la duración a la apertura del instante...

\section{Proposiciones lógico-operativas}

Para alcanzar a la identificación de las bases que permitan la reformulación del planteamiento ambiental, hace falta lograr una síntesis propositiva que permita llegar a una mayor claridad axiológica y epistemológica. Para ello se exponen a continuación las siguientes proposiciones lógicooperativas que tienen especial relevancia para la reformulación del ambiente sobre bases holístico-minimalistas.

\section{Proposición 1}

El ambiente estructuralmente hablando es el conjunto compuesto por las interrelaciones entre los elementos físico-químicos, bióticos, sociales y culturales.
De manera operativa, y sobre el sustento de la lógica de conjuntos, por cuanto esta permite mantener la visión holística aún reconociendo la existencia de las partes; este enunciado propositivo tiene las siguientes implicaciones:

Siendo A un conjunto ambiental determinado, FQ representa el subconjunto de elementos físico-químicos, B el subconjunto de los elementos bióticos, $\mathrm{S}$ el subconjunto de los elementos sociales y $\mathrm{C}$ el subconjunto de los elementos culturales. Sin embargo, una condición indispensable para que cada subconjunto de elementos sea considerado como parte del conjunto A es que esté interrelacionado con los otros subconjuntos estructuralmente identificados, es decir, que:

$$
\begin{aligned}
& F Q \varepsilon A \rightarrow A=\{F Q \cap(B \cup S \cup C)\}<->A=\{(F Q \cap B) \cup(F Q \cap S) \cup(F Q \cap C)\} \\
& B \varepsilon A \rightarrow A=\{B \cap\{F Q \cup S \cup C)\}<->A=\{(B \cap F Q) \cup(B \cap S) \cup(B \cap C)\} \\
& S \varepsilon A \rightarrow A=\{S \cap\{F Q \cup B \cup C)\}<->A=\{(S \cap F Q) \cup(S \cap B) \cup(S \cap C)\} \\
& C \varepsilon A \rightarrow A=\{C \cap\{F Q \cup B \cup S)\}<->A=\{(C \cap F Q) \cup(C \cap B) \cup(C \cap S)\}
\end{aligned}
$$

Esta propiedad distributiva del álgebra de conjunto está indicando la existencia de una estructura policéntrica en el ambiente.
Para comprender mejor este planteamiento, en la figura 7 se realiza su expresión gráfica.

FIGURA 7 - EL POLICENTRISMO AMBIENTAL

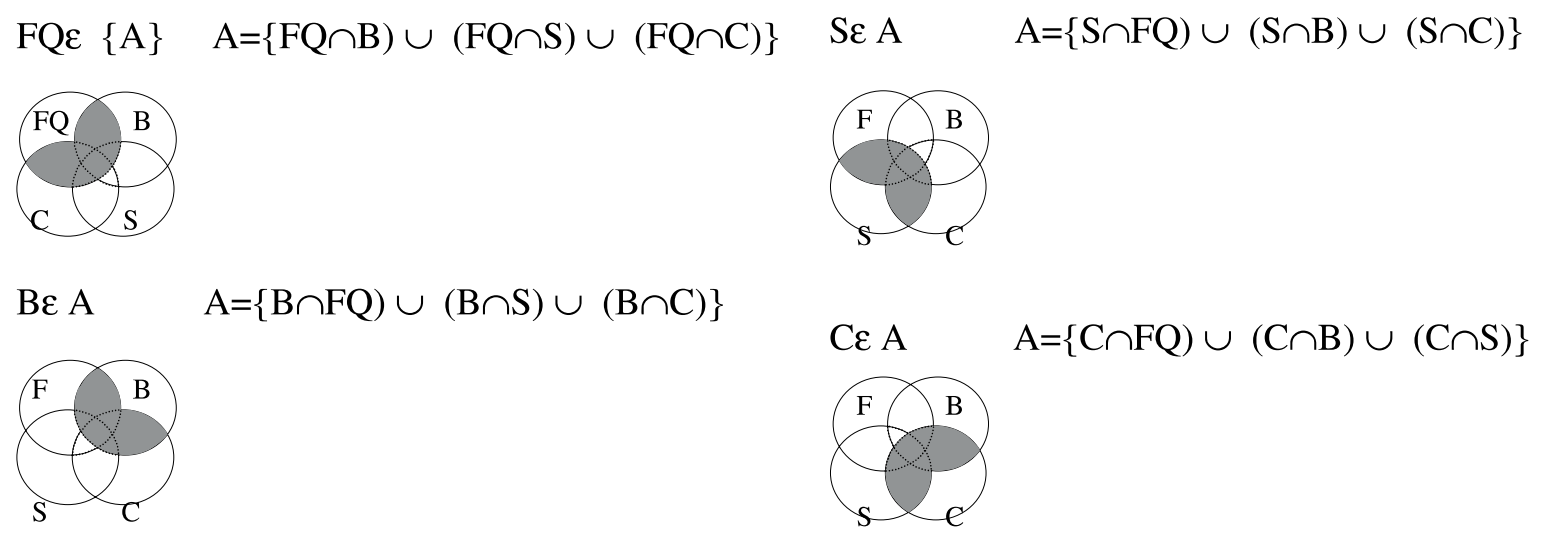


Esta consideración gráfica, también permite una expresión más rigurosa mediante una tabla binaria de verificación, que muestra las distintas posibilidades de distribución de un elemento cualquiera del conjunto $\{\mathrm{A}\}$ en los distintos subconjuntos que lo conforman (tabela 1).

\section{TABELA 1 - VALIDACIÓN DEL POLICENTRISMO AMBIENTAL}

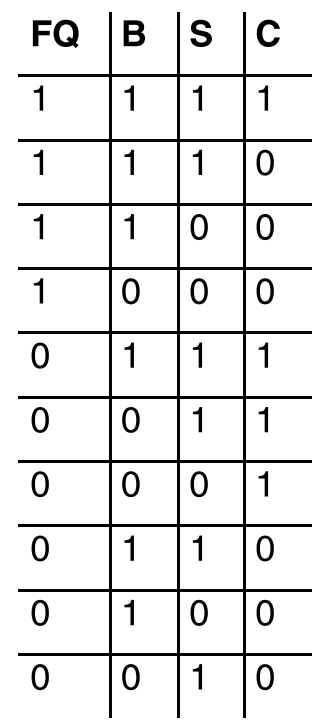

1: aparece en el subconjunto.

0: no aparece en el subconjunto.

Lo importante de este cuadro es que muestra las diferentes opciones que tiene un elemento de aparecer en el conjunto $\{\mathrm{A}\}$ en función de su distribución en sus distintos subconjuntos. Esta diversidad de situaciones conlleva a aceptar que de considerarse la existencia de las partes en el todo ambiental, la estructura de un conjunto ambiental dado, no necesariamente debe presentar una distribución estructural equitativa y equiprobable de cada uno de los elementos constitutivos a lo largo de los subconjuntos que lo conforman. Son mayores las posibilidades de que esta distribución esté sesgada hacia algunos de los subconjuntos, convirtiéndose por lo tanto esta desigualdad en la norma más que en la excepción.

A manera de conclusión, interpretando los resultados de la aplicación de los círculos de Ven y de la Tabla de Validación, se puede afirmar que a pesar de mantener como constante la tetraestructura del ambiente, dependiendo del subconjunto que se utilice como centro, surgen diferencias en cuanto a la demarcación del ámbito de las intersecciones a considerar. El policentrismo ambiental es una derivante de la aceptación del postulado del holismo minimalista, por cuanto tan solo mediante la existencia de la diversidad en la unidad ambiental, se puede reconocer el peso diferencial de cada parte en el todo.

Estas consideraciones de orden epistemológico y lógico, están en concordancia con lo que los investigadores del campo ambiental han venido reconociendo en la práctica como un gradiente en los niveles de naturalidad o de antropización (depende del criterio bien naturalista o bien culturalista que se escoja) de las diferentes unidades del umwelt o mundo ambiental.

Es importante destacar que esta posición holística minimalista también permite superar la especie de enciclopedismo ambientalista basado en la premisa de que "todo esta relacionado con todo" (COMMONER, B. 1972), tan en boga a partir de los años '70. De aceptar este postulado en lugar de intersección $(\cap)$ entre conjuntos se estaría hablando de unión $(\cup)$ entre conjuntos, lo cual son dos situaciones, si bien no antagónica si bastante distintas, como se expresa gráficamente en las figuras 8 y 9.

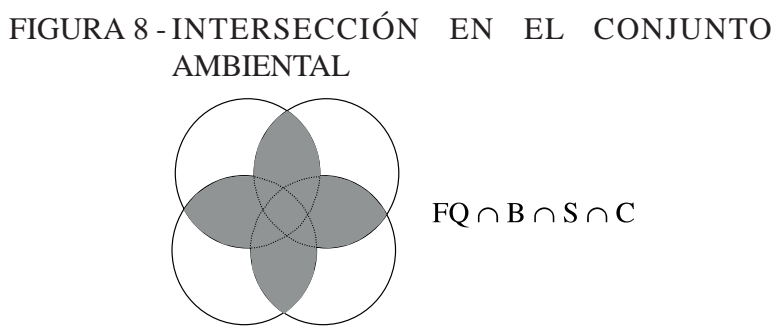

FIGURA 9 - UNIÓN EN EL CONJUNTO AMBIENTAL

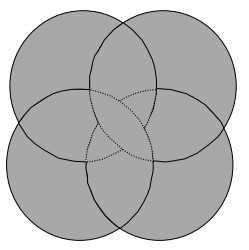

$\mathrm{FQ} \cup \mathrm{B} \cup \mathrm{S} \cup \mathrm{C}$

Para el planteamiento ambiental integral que intenta consolidarse es importante tener presenta esta distinción entre intersección y unión. El resto de los elementos que no participan en la intersección tetraédrica general deben ser tomados como complemento A' del conjunto ambiental A. 


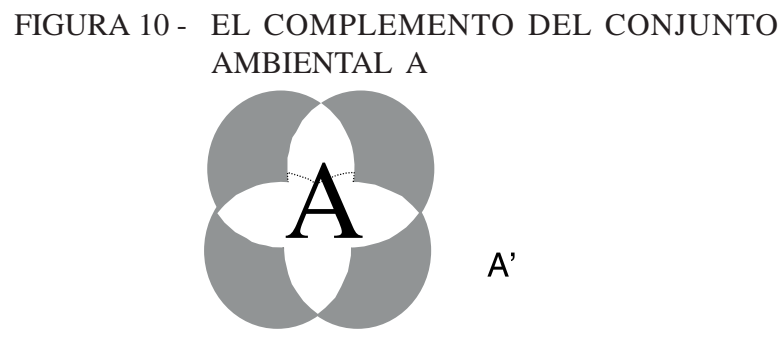

Con ello se reconoce que las intersecciones entre los subconjuntos solos abarcan una parte de los mismos, el complemento restante queda como un marco de referencia contextual, que se incorpora al análisis en la medida en que permite interpretar las interrelaciones que le dan razón de ser al conjunto A.

Esto lleva a establecer que la intersección que da cuenta de lo ambiental es válida tan sólo para algún $\mathrm{x}$ (эx) y no para todo $\mathrm{x}(\forall \mathrm{x})$.

De manera general, un conjunto ambiental A se puede expresar algebraicamente como: $\mathrm{A}=\{\ni \mathrm{x} F Q \cap \ni \mathrm{x} B \cap \ni \mathrm{x}$ $\mathrm{S} \cap \ni \mathrm{x} C\}$.

La intersección se convierte por lo tanto en la operación que expresa algebraicamente el significado de las interrelaciones e interacciones como procesos básicos de la organización ambiental .

A manera de enunciado lógico verbalizado, tenemos que: sea $\mathrm{A}$ un ambiente concreto, su estructura se representa como: $A=\{\ni x F Q \cap \ni x B \cap \ni x S \cap \ni x C)$, es decir como el producto de la intersección entre: $\mathrm{FQ}$ (A) o el subconjunto de los elementos bióticos que están en interrelación con los restantes subconjuntos de $\{\mathrm{A}\}$; B (A) o el subconjunto de los elementos bióticos que están en interrelación con los restantes subconjuntos de $\{\mathrm{A}\} ; \mathrm{S}$ (A) o el subconjunto de los elementos sociales que está en interrelación con los restantes subconjuntos de $\{\mathrm{A}\}$; y $\mathrm{C}$ (A) o el subconjunto de los elementos culturales que está en interrelación con los restantes subconjuntos de $\{\mathrm{A}\}$.

\section{Proposición 2}

Las organizaciones ambientales son estructuras espacios-temporales resultantes del acoplamiento de componentes con especialidades y temporalidades diferenciales. Entonces:

$A=\{\ni x F Q(e, t) \cap \ni x B(e, t) \cap \ni x S(e, t) \cap \ni x C(e, t)\}$
El espacio-tiempo del conjunto ambiental es el resultado del acoplamiento entre los espacios-tiempos propios de cada componente.

Si esto se vincula con el policentrismo de la proposición 1 , se tiene que la centralidad de un componente en una determinada estructura ambiental, está asociada también a la necesaria supeditación de las temporalidades y espacialidades de los restantes componentes al espacio-tiempo de los componentes seleccionados como centro. Llevando este planteamiento al caso de la cultura, es decir, de acuerdo a la convencionalidad ambientalista, el nivel más alejado de la "naturaleza", en los conjuntos ambientales centrados en las actividades de la especie humana, el acoplamiento o desacoplamiento que dan carácter a la estructura están supeditados a su medición, calificación y valoración en la escala de tiempo humano, en cualquiera de sus dimensiones: psicológica o personal subjetiva; objetiva o impersonal, socialmente convenida, biológica. Si bien el desconocimiento de las otras temporalidades ambientales podría causar la destrucción de estas estructuras centradas en la cultura, la consideración de las mismas no necesariamente determina la sobre vivencia del conocimiento y las instituciones que identifican al nivel cultural. De manera preposicional específica se puede expresar la situación planteada de la siguiente manera: $\mathrm{P} \Lambda \mathrm{Q}$.

Siendo P la proposición A que establece que el desconocimiento de las temporalidades de subconjuntos ambientales, físico, químicos, bióticos y sociales, pertenecientes ( $\ni$ ) a un conjunto $\{\mathrm{A}\}$ centrado en la cultura, podría causar la destrucción de estas estructuras, se presenta una situación de conjunción con una propuesta que establece que el conocimiento de estas temporalidades no culturales no garantizaría necesariamente la perdurabilidad cultural. En términos generales se considera que las temporalidades de los subconjuntos evolutivamente anteriores a un subconjunto dado requieren ser tomadas en cuenta para evitar la destrucción del subconjunto en cuestión. Sin embargo la permanencia del mismo está supeditada a su propia dinámica, es decir a las pautas temporales que le son inherentes. Así, la evolución biológica requiere del tiempo geológico para demarcar su desempeño en términos del devenir del planeta, la historia humana requiere del marco temporal biológico y geológico para establecer sus posibilidades de permanencia como especie. Sin embargo, así como la evolución de la vida, está fundamentalmente vinculada a los procesos propios de este nivel considerados en el ideograma y no de los procesos propios del nivel físico-químico, la cultura, sus posibilidades de permanencia, de transformación, se 
sustentan en los mecanismos considerados propios de este nivel y no en el biológico, o en el físico-químico, ni siquiera en el social.

Con respecto al espacio, al igual que con el tiempo existen expresiones diferenciales de espacialidad para los distintos componentes: desde la planetaria de los ciclos biogeo-químicos hasta la microscópica de los átomos y células. En la esfera cultural están presentes todas estas espacialidades, sin embargo condicionadas a la métrica y simbolización que identifica la espacialidad humana. Igual que en el caso del tiempo, el no tomar en cuenta las espacialidades no humanas podrían menoscabar el devenir de las estructuras ambientales con centro en lo cultural, pero la sobrevivencia de las mismas no está garantizada por la consideración de las espacialidades físico-químicas, bióticas y sociales distintas a la humana.

En la medida en que se acepta que existen realidades diferentes de acuerdo a la centralidad del subconjunto ambiental que se esté considerando, se debería admitir que los acoplamientos temporales y espaciales deben propiciarse de acuerdo a los valores propios del conjunto de referencia.

De tal manera que la necesidad de acoplamientos espacios-temporales -, lleva a plantear la necesidad de la dimensión informacional propia de cada nivel, en función de la cual se propician los encuentros y desencuentros funcionales o temporales -, y morfológicos o espaciales, de acuerdo a los diversos códigos de información ambiental.

Como se consideró en el ideograma,la cultura posee un tipo de información que le es propia, que es la semántica cultural basada en los códigos que permiten establecer la comunicación y control sobre la búsqueda y configuración de las diferentes formas y conocimientos y de la permanencia de las instituciones. En la información semántica cultural es donde se deben buscar las claves que permiten determinar los acoplamientos funcionales y morfológicos, mediante los cuales se logren introducir las expresiones temporales y espaciales de los restantes componentes ambientales, necesarios para la sobrevivencia de las estructuras humanas. Esto se podría enunciar de la siguiente manera:

Puede persistir toda estructura ambiental centrada en el subconjunto cultural si la información inherente que la controla y comunica garantiza el acoplamiento funcional y estructural de los componentes humanos con los restantes componentes.

\section{Proposición 3}

La permanencia de una organización ambiental es una probabilidad, por lo tanto las estructuras ambientales deben ser consideradas como organizaciones fluctuantes, es decir con variaciones espacios-temporales que indican estados oscilantes.

Esto conduce a plantear la cuestión de la entropía y neguentropía en el intercambio energético ambiental. Al respecto se debe recordar que las organizaciones ambientales están supeditadas al igual que todo el planeta a las leyes de la termodinámica. Es decir que para el umwelt en su conjunto la energía no se crea ni se destruye, se transforma (I Ley de la termodinámica) y la energía que la dinamiza tiende a su transformación irreversible en calor o en energía no útil (II Ley de la termodinámica).

En cuanto al primer enunciado, la manera como se concibe la dinámica ambiental teniendo como proceso conductor la cascada de energía electromagnética solar, resulta suficientemente explicativa. El punto a resolver, se centra en el segundo principio, por cuanto ¿cómo se puede hablar de organización si la tendencia de la energía, su estado más probable es la desorganización entrópica? Este es uno de los dilemas cruciales de un debate el que todavía no existe. Conclusiones definitivas, sin embargo, a continuación se intentan exponer por lo menos la posición personal sobre algunos puntos críticos de interés para la comprensión energética termodinámica del ambiente.

En este sentido, se debe empezar por decir que para el ambiente en su conjunto se generalizan las consideraciones que sobre la vida como manifestación neguentrópica se han venido haciendo a partir del trabajo de Schoridenger (1967). Esta es una premisa sobre la que en términos generales existe acuerdo. De tal manera que el ambiente, su organización, se convierte en una especie de retardo de la degradación energética. Toda organización ambiental se convierte en una "isla" de orden, en el universo del proceso de desorganización termodinámica de la energía. De tal forma que, en la tendencia macroscópica del universo y del planeta a la degradación termodinámica, el umwelt terrestre se convierte en una organización macroscópica neguentrópica. De acuerdo a la termodinámica estadística de Boltzman, la entropía de un sistema termodinámico es una función del número de arreglos microscópicos de los elementos constitutivos. En la medida en que el número de estados microscópicos de 
un sistema determinado sea menor, menor también es la entropía macroscópica del sistema en su conjunto. De manera inversa, en la medida en que aumenta el número de micro-estados aumenta el desorden del sistema (FENZL, 1996).

En términos de análisis combinatorio, esta situación se sintetiza en la fórmula:

$$
\mathrm{P}=\frac{\mathrm{N} !}{\mathrm{n} 1 ! \ldots \mathrm{n} 2 !}
$$

donde $\mathrm{P}$ expresa el número de maneras de realizar una repartición particular en los estados $n 1, n 2$ de $\mathrm{N}$ elementos constitutivos del sistema. Si en lugar de dos estados se tuviesen k estados con n, la fórmula general es:

$$
\mathrm{P}=\frac{\mathrm{N} !}{\Pi \mathrm{n} !}
$$

En términos generales, $\mathrm{P}$ define el número de micro estados posibles para realizar un macro estado del sistema. En la medida en que las diferencias en la distribución de $\mathrm{N}$ son menores el valor de $\mathrm{P}$ aumenta. Es decir que en un sistema dado en la medida en que existe una mayor homogeneidad en la distribución de los elementos en los distintos subconjuntos mayor es $\mathrm{P}$ y por lo tanto mayor es la entropía del sistema.

Boltzman tradujo el cálculo de $\mathrm{P}$ en términos de entropía del sistema, mediante la fórmula: $\mathrm{S}=\mathrm{k}$ In $\mathrm{P}$, dado que siendo el número de complexiones $\mathrm{P}$ un valor multiplicativo, y la entropía un valor aditivo, se toma el logaritmo neperiano de $\mathrm{P}$ para de esta forma hacer equivaler $\mathrm{P} 1+2=\mathrm{P} 1$. P2 k. De manera relacionalmente sintética se puede afirmar que:

1) El desorden de un microestado se mide ordinalmente por la probabilidad del macroestado correspondiente.

2) El desorden y la probabilidad de un macroestado son proporcionales al número de microestados correspondientes “...por lo tanto se está en la capacidad de decir que conociendo la repartición de los elementos se conoce cuál es el grado de desorden de un sistema" (FORSÉ, 1989, p. 75).
La aceptación de estos enunciados pondría en entredicho la posibilidad de mantener sistemas ambientales neguentrópicos en el umwelt, por cuanto, una tendencia generalizada de toda organización ambiental, tanto predominantemente natural como predominantemente cultural, es la diversificación, es decir el aumento del número de microestados y de elementos, y por lo tanto de los niveles de entropía. Por consiguiente, este aumento en lugar de convertir a las organizaciones ambientales en estructuras neguentropicas, en realidad tan solo estaría retardando su proceso entrópico. No se estaría más que alargando el tiempo de muerte termodinámica. Sin embargo, de ser esto último cierto, no se entiende el surgimiento a lo largo de los procesos de emergencias, de novedades, de creación. La muerte termodinámica, contrariamente se circunscribe a la repetición constante. Es por ello fundamental, en el caso de la diversidad, diferenciar entre el aumento del número de elementos y el incremento de los tipos o macroestados de elementos. Esta consideración conduce a la necesidad de considerar a las organizaciones ambientales como sistemas abiertos para los cuales la neguentropía se mantiene como búsqueda constante del orden a expensas del desorden del contexto con el que interactúa. De acuerdo a Prigogine y Stenger (1979; 1988), la relación entropía-neguentropía en los sistemas abiertos se explica mediante la fórmula:

$$
\text { Ds }=\text { Des }+ \text { Dis }
$$

donde:

Ds = variación de la entropía

Des $=$ el flujo de entropía entre el sistema y su contexto

Dis = entropía producida, es decir creada inevitablemente en el interior del sistema

En esta expresión, Dis es siempre positiva o nula e indica que el sistema, que la entropía del sistema tiende aumentar o a mantenerse constante. Este estado de equilibrio lo alcanza el sistema a expensa de su contexto, es decir que, para que un sistema se mantenga organizado, debe aumentar al grado de desorganización de su contexto. Es esta la equivalencia básica a tenerse en cuenta en función de la II Ley de la termodinámica, que en definitiva no niega que existan excepciones locales de neguentropía, y de estas ocurrir no negaría tampoco la tendencia general a la degradación de la energía total del conjunto sistema + contexto. Sin embargo queda por resolver que tan frecuentemente pueden ocurrir las excepciones neguentrópicas. 
En términos de termodinámica lineal, es decir de sistemas en equilibrio o cercanos al equilibrio, estas excepciones son poco probables, por cuanto la necesidad de mantener el equilibrio anula prácticamente toda posibilidad de novedad. Sin embargo, en términos de termodinámica no lineal de los sistemas alejados del equilibrio, las posibilidades aumentan por cuanto, la inestabilidad constante, permite la aparición de fluctuaciones que podrían, en función de su intensidad introducir novedad en el comportamiento del sistema, produciéndose una bifurcación comportamental.

De tal manera que al orden por mantenimiento del equilibrio lineal dirigido a comprender la estabilidad recurrente de los sistemas se le antepone el orden por fluctuación, dirigido a comprender como una situación de inestabilidad no recurrente puede conducir a una reestructuración del sistema.

\section{Implicaciones epistemológicas de las proposi-} ciones desarrolladas

Las proposiciones realizadas sobre el policentrismo ambiental, la diversidad espacio-temporal, el orden fluctuante desde una visión personal, tiene profundas repercusiones para el planteamiento ambiental convencional, sustentado en la interpretación cósmico-planetariaevolutiva. Para mostrar el alcance de las mismas, tomemos como objeto de análisis la ciudad. Esta se convierte en el mundo contemporáneo en la forma de hábitat que la humanidad en su conjunto está privilegiando. La población urbana en el ámbito mundial está creciendo de manera sostenida, a una tasa del 2,5\% interanual, cifra que prácticamente duplica la rata de aumento demográfico general del mundo calculada en 1,3\% (UNFPA, 2000). En la actualidad alrededor del cincuenta por ciento de la población mundial está viviendo en aglomeraciones urbanas de distintos tamaños y densidades.

Convencionalmente hablando la ciudad se ha utilizado como una especie de negación de lo ambiental. Los ambientalistas y ecologistas, parecieran que han intentado reproducir entre la ciudad y en el ambiente la antitesis hombre-naturaleza. La ciudad se convierte en el marco del planteamiento evolucionista, en el punto mínimo de presencia de componentes naturales. Sin embargo al asumirse posiciones mas abiertas, dándose cabida al policentrismo ambiental, la ciudad se convierte en el máximo de posibilidades para humanizar el medio natural en función de la capacidad del hombre como ser transformador del ambiente "Al igual que un arrecife coralino, una colmena o un territorio, la ciudad es a un tiempo soporte y consecuencia del organismo social que vive en su seno" (ROSNAY, apud VIDART, 1986, p. 65), recordando por supuesto que el hombre hace parte de la naturaleza y, mediante la cultura, se convierte en una manifestación particular del ambiente. Así como los medios propios del nivel físico-químicos se diluyen en el biotopo para garantizar una lectura desde lo biológico, en una interpretación desde la cultura, como es la que se exige para la ciudad, el antrotopo emerge como noción envolvente del biotopo y por ende de los medios físico-químicos. Por supuesto que en una interpretación de este tipo se mantiene el vínculo estructural entre los distintos niveles ambientales, eso sí evitándose las posturas reduccionistas naturocéntricas.

Se debe recalcar también que en el marco de una postura policéntrica, igualmente se considera el aspecto de concatenación en el tiempo de los hechos, sin embargo más que la supeditación a la causa primera, se intenta interpretar los fenómenos como instantes que emergen del flujo energético con características propias. En el caso de la cultura y de una de su expresión más emblemática, como lo es la ciudad, a pesar de mantenerse los condicionantes básicos físico-químicos, bióticos y sociales de referencia para el conjunto de la biosfera, estos por sí solo no son suficientes para permitir la comprensión de la cultura como hecho relevante en el ambiente. Por ejemplo, para entender el significado de las instituciones en las relaciones hombre-ambiente, se debe buscar mas en el nivel propiamente cultural de la noosfera, que en la misma biosfera. De manera similar, para comprender el papel de las distintas formas de conocimiento en el ambiente, la génesis del planeta se convierte en un dato referencial, pero no sustancial, debiéndose insistir más en el reconocimiento de las claves que han permitido la conformación de las religiones, de las diferentes manifestaciones artísticas, de la ciencia como institución, es decir en las distintas manifestaciones en las que se expresan el potencial del hombre como ser pensante y transformador, adaptativo o destructivo, de la naturaleza.

Las consideraciones sobre el reduccionismo del tiempo evolutivo en estudios ambientales deben complementarse en el contexto propositivo anteriormente enunciado, con los referentes termodinámicos a tener en cuenta en el estudio de la diversidad de las organizaciones que conforman la unidad ambiental. Continuando con el 
caso de la ciudad, las diversas orientaciones que intentan su interpretación ambiental - la ciudad como hábitat humano, la ciudad como ecosistema fallido y/o incompleto, la ciudad sustentable, la ciudad del ecodesarrollo, entre otros (LISIO, 2000) - por lo general se dirigen, desde el punto de vista funcional, a la búsqueda del equilibrio y la estabilidad. Es decir, a través de estas posturas, se estaría aceptando que las organizaciones urbano ambientales están en una situación de equilibrio lineal, que además en el mejor de los casos estaría manteniendo su entropía interna: nula, sin aumento, pero sin disminución. Sin embargo, en el marco del planteamiento de las estructuras de equilibrio oscilante, el estudio ambiental de la ciudad al igual que cualquier otra organización ambiental caracterizada por el policentrismo y por lo tanto la desigualdad en la distribución de sus elementos constitutivos, debería estar dirigido a la comprensión de las situaciones de desequilibrio. Estas son las que permiten comprender a las organizaciones ambientales, en este caso urbanas, como sistemas en equilibrio metaestable, que pueden disminuir su entropía interna, por supuesto siempre a expensas del aumento en la entropía de su contexto, y con ello no contradiciendo los postulados básicos de la segunda ley de la termodinámica.

En el mundo real, la ocurrencia de efectos "bola de nieve", tanto en el marco del nivel físico-químico como del biológico y hasta del cultural, permite evidenciar los casos de no linealidad. Sin embargo, se ha preferido, por lo general, asumir que la estabilidad se logra en función del equilibrio de la anulación de las fuerzas reconocidas, más que en función de procesos no esperados, desapercibidos o sencillamente y escasamente evaluados.

\section{Algunos comentarios finales}

El policentrismo y la termodinámica no lineal conducen a plantear la diversidad de los marcos espaciostemporales de las organizaciones ambientales que están oscilando en tanto que sistemas abiertos entre la entropía del contexto y la neguentropía interna. En el marco del ejercicio de axiomatización y reflexión epistemológica que se ha intentado presentar, se puede definir una tipología elemental de las organizaciones ambientales, que, manteniendo la estructura tetracéntrica básica, permite clasificarlas en:

- conjuntos ambientales centrados en los factores físico-químicos;
- Conjuntos ambientales centrados en los factores bióticos;

- Conjuntos ambientales centrados en los factores sociales;

- Conjuntos ambientales centrados en los factores culturales.

Decir que un subconjunto sea predominante en una organización ambiental tan solo ayuda a la diferenciación de los elementos que permiten establecer la centralidad y direccionalidad de la estructura espacial y funcionamiento temporal del conjunto, pero no permite la calificación de los mismos. En otras palabras, el hecho de que una organización ambiental esté centrada en la cultura no la hace ni mejor ni peor que por ejemplo una organización centrada a los factores bióticos. A pesar de que la ortodoxia ambientalista prejuzgue como positivo la predominancia de los componentes físico-químicos y sobre todo bióticos, en realidad no es cantidad de naturaleza o de cultura lo que determina la viabilidad de las organizaciones ambientales, sino la manera como los distintos tipos de componentes se interrelacionan espaciotemporalmente para el mantenimiento de la organización. Lo ambiental, más que una cuestión de cantidades y proporciones, es un problema de calidad de vínculos. El problema ambiental de las organizaciones predominantemente culturales, como las ciudades, en su esencia no se resuelve incrementando la presencia de los componentes naturales ya que el quid de la cuestión está en la articulación y/o desarticulación de la trama de interrelaciones sobre la que descansan los vínculos intercomponentes. Es por ello que para calificar una determinada organización ambiental hace falta bajar al nivel más operativo de los acoplamientos y desacoplamientos en el espacio y en el tiempo entre los componentes de la organización, debiéndose tener presente que los postulados de la termodinámica no lineal o de las organizaciones abiertas alejadas del equilibrio indican que toda realidad que se interpreta como sistema tiene una historia propia, que demarca un recorrido particular entre un conjunto de opciones (PRIGOGINE; STENGER, 1979; 1988). Las dimensiones espacios-temporales basadas en la apertura policéntrica permiten calificar autorenferenciadamente, es decir en función de comportamientos particulares, a las organizaciones ambientales, sin necesidad de utilizar supuestos universales que impiden captar emergencia de lo local y del instante. 
LISIO, A. de. Del determinismo de la duración a la apertura del instante...

\section{REFERENCIAS}

BEAUREGARD, C. Le second principe de la science du temp. Paris: Ed. du Seuil, 1963.

BERTALANFFY, L.V. Teoría general de sistemas. México: Fondo de Cultura Económica, 1976.

BRICEÑO, R. La naturalización de lo social. In: ACOSTA M.; BRICEÑO, R. Ciudad y capitalismo. Caracas: Ediciones de la Biblioteca U.C.V., 1987. p. 79-98.

BOOCKCHIN, M. Los limites de la ciudad. México: Nueva Imagen, 1974.

Toward an Ecological society. Black Rose. 1980.

From urbanization to cities. Towards a new politics of citizenship. [S.1.]: Vermont, 1995.

BUCKLEY, W. La sociología y la teoría moderna de sistemas. Buenos Aires: Amorrortu, 1974.

BUNGE, M. El principio de la causalidad en la ciencia moderna. Buenos Aires: Ediciones Universitarias, 1956.

CREESE, W. The search for environment. The garden city. Before and after. Baltimore: The John Hopkins University Press, 1992.

DANSERAU, P. Essay de classification et de cartograaphie ecologique des espaces. Québec: Universite Laval, 1985.

LISIO, A. de. Entropía y neguentropia urbanas. Bases para la reformulación del estudio ambiental de la ciudad. Venezuela, 1999. PhD Thesis - Facultad de Arquitectura y Urbanismo de la Universidad Central de Venezuela. Mimeog.

DESCARTES, R. 1983 Discurso del método. Reglas para la dirección de la mente. Barcelona: Orbis, 1983.

DION, E. Invitation a la Theorie de I'information. Paris: Edition du Seuil, 1997.

FEYERABEN, P. Tratado contra el método. Madrid: Tecnos, 1981.

FORSÉ, M. L'ordre improbable. Entropía et processus social. Paris: Press Universitaire de France, 1989.

. L'analyse structurrelle du changement sociale. Le modele de Louis Drin. Paris: Press Universitaire de France, 1991.

Le reseaux sociaux. Paris: Armand Colin, 1994.

GONZÁLEZ, A. R. et al. El IDEOGRAMA Ambiental. Caracas: [s.n.], 1984. (Serie Cuadernos del Cenamb, n. 840).
HARVEY, D. Explanation in geography. [S.1.]: Eduard Arnold, 1969. 1977.

Urbanismo y desigualdad social. Madrid: Siglo XXI,

KHUN, T. 1975 La Estructura de las revoluciones científicas. México: Fondo de la Cultura Económica, 1975.

LABORIT, M. El hombre y la ciudad. Barcelona: Kairos, 1972.

LAKATOS, I. La historia de la ciencia y sus reconstrucciones naturales. Madrid: Tecnos, 1981.

LAVELOCK, J. Les ages de Gaia. Paris: Robert Laffont, 1990.

LINDEMAN, R. The trophic - dynamic aspect of ecology. Ecology, v. 23, n. 4, p. 399-418, 1942.

MACHLIS, G.; FORCE, J. E.; BURCH, W. The human ecosystem. Part 1: the human ecosystem as an organization concept. ecosystem management. Society \& Natural Resources, v. 10, n. 4, p. 347-368, 1997.

Mc. KAY, D. Information, mechanism and meaning. Cambridge: MIT, 1969.

MONOD, J. El azar y la necesidad. Ensayo sobre la filosofía natural de la biología. Caracas: Monte Ávila, 1971.

MORA, F. Diccionario de Filosofía. [S.1.]: Blume, 1978.

MORIN, E. Le paradigme perdú. Paris: Press Universitaire de France, 1971.

La methode. La nature de la nature. Paris: Editions du Seuil, 1978. 1982.

La methode. La biologie. Paris: Editions du Seuil, 1984.

Ciencia con conciencia. Barcelona: Editorial Anthropos,

La methode. La connaiscence de la connaiscence. Paris: Editions du Seuil, 1986.

OBADIA, G. N. L'espace originel. Berne: Peter Lang, 1984.

ODUM, H. T. Ecology systems. New York: John Willey and Sons, 1983

PRIGOGINE, I.; STENGER, I. La nouvelle alliance. Methamorphose de la science. Paris: Gallimad, 1979. 
LISIO, A. de. Del determinismo de la duración a la apertura del instante...

Entre le temp et l'eternel. ED. Flammarion, 1988.

RAPAPORT, A. 1978 Aspectos humanos de la forma urbana. Barcelona: Gustavo Gili, 1978.

REES,W. Ecological foot prints and appropriated carrying capacity: what urbans leaves out. Environment and urbanization, v. 4, n. 2, p. 121-130, 1992.

REEVES, H. et al. Synchronicite I' ame et la science. Existe - t- il un ordre a - causal? Paris: Poiesis, 1984.

DE ROSNAY, J. El macroscopio. Hacia una visión global. Madrid: Editorial AC, 1976.

SACHS, I. Strategies de I' ecodeveloppement. Paris: Ouvriers, 1981.

SCHORINDINGER, F. What is life? Boston: Cambridge University Press, 1967.
TANSLEY, G. The use and abuse ot vegetation concepts and terms. Ecology, v. 16, n. 3, p. 284-307, 1936.

TAYLOR, M. Industrialism, enterprise, power and environmental Change. Environment and planning, v. 28, n. 6, p. 1035-1052, 1996.

VIDART, D. Filosofía ambiental. Epistemología, praxiologia didáctica. Bogotá: Nueva América, 1986.

WEBER, M. The urban place and the non place urban realm. In: Weber, M. et al. Exploration into urban structure. Philadelphia: University of Pennsylvania Press, 1964. p. 79-153.

WORSTER, D. Le pioners de l'ecologie. Paris: Press Universitaire de France, 1992. 